Technical Memorandum 33-524

\title{
The Response of Covered Silicon Detectors to Monoenergetic Gamma Rays
}

\author{
Melvin Reier
}

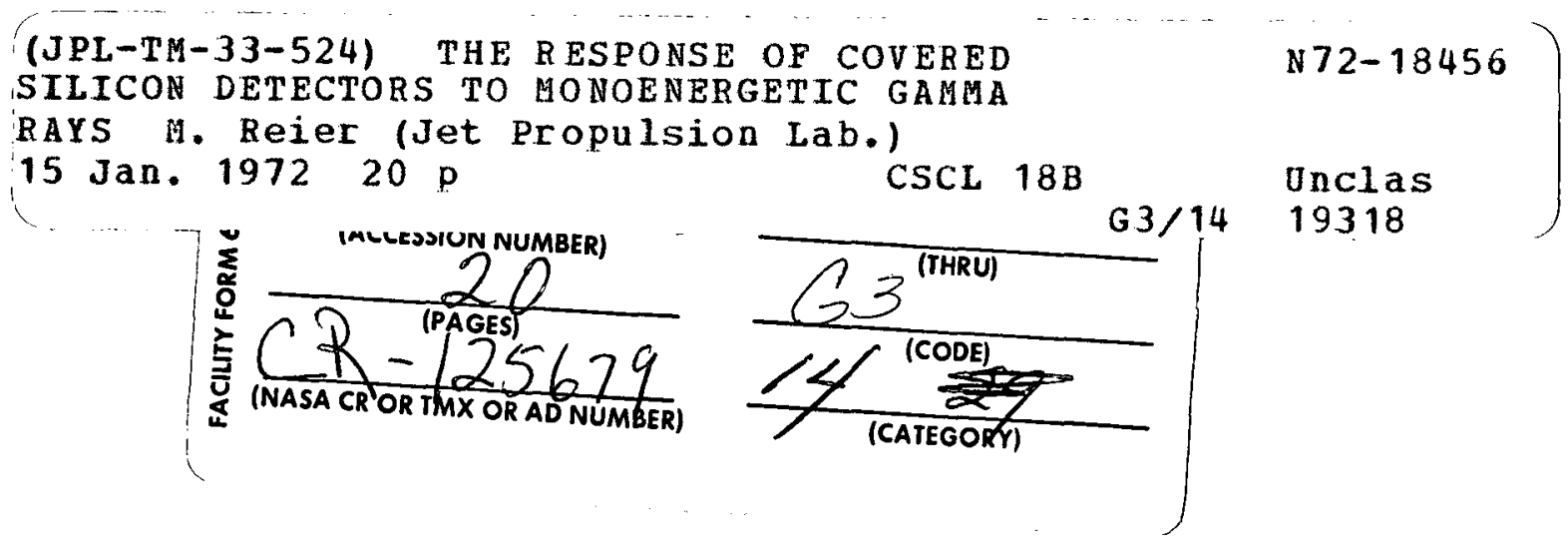

\footnotetext{
JET PROPULSION LABORATORY

CALIFORNIA INSTITUTE OF TECHNOLOGY

PASADENA, CALIFORNIA
}

January 15,1972

Reproduced by 
NATIONAL AERONAUTICS AND SPACE ADMINISTRATION

\title{
Technical Memorandum 33-524
}

\section{The Response of Covered Silicon Detectors to Monoenergetic Gamma Rays}

\author{
Melvin Reier
}

JET PROPULSION LABORATORY

CALIFORNIA. INSTITUTE OF TECHNOLOGY

PASADENA, CALIFORNIA

January 15,1972 
Prepared Under Contract No. NAS 7-100 National Aeronautics and Space Administration 
TECHNICAL REPORT STANDARD TITLE PAGE

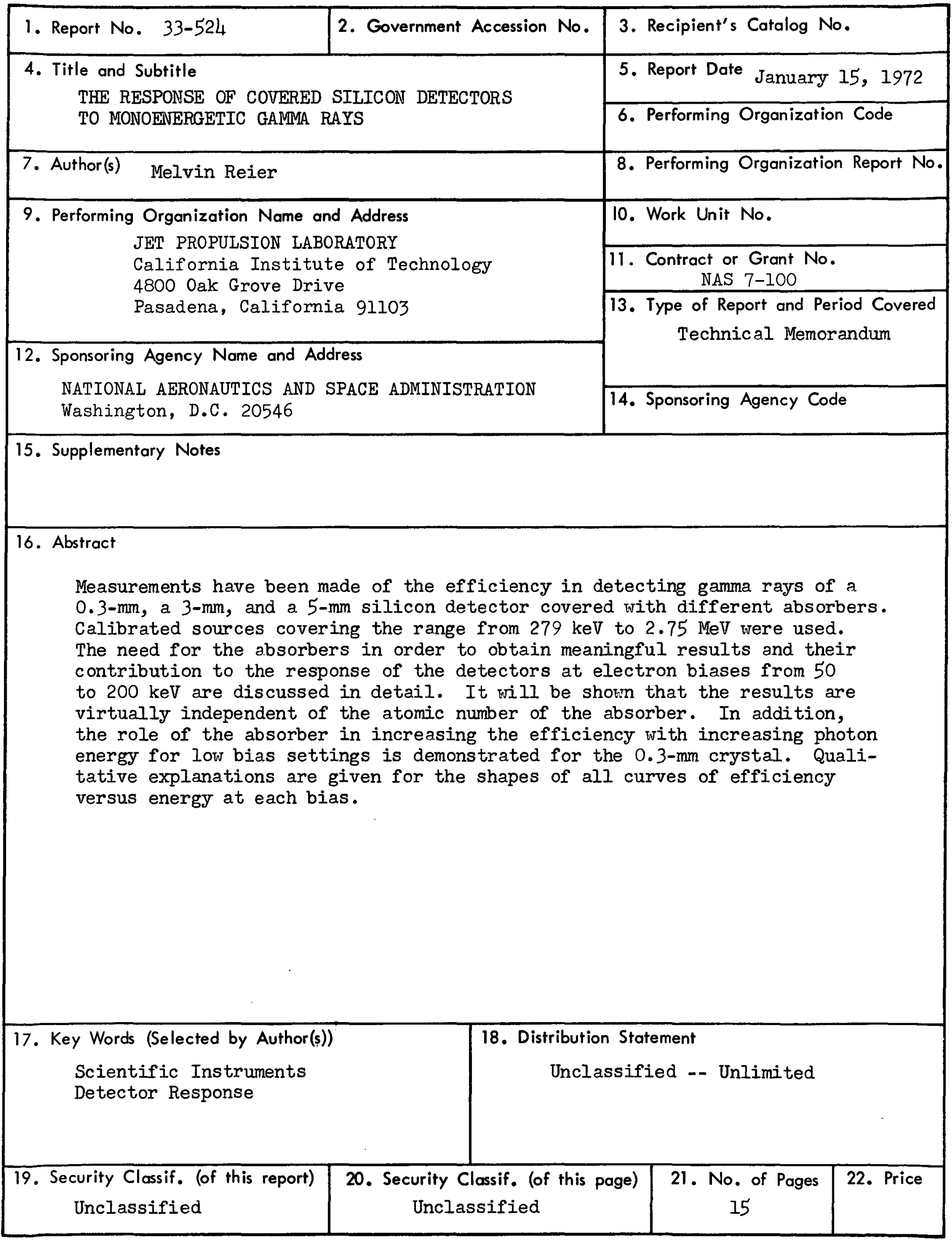




\section{HOW TO FILL OUT THE TECHNICAL REPORT STANDARD TITLE PAGE}

Make items 1, 4, 5, 9, 12, and 13 agree with the corresponding information on the report cover. Use all capital letters for title (item 4). Leave items 2, 6, and 14 blank. Complete the remaining items as follows:

3. Recipient's Catalog No. Reserved for use by report recipients.

7. Author(s). Include corresponding information from the report cover. In addition, list the affiliation of an author if it differs from that of the performing organization.

8. Performing Organization Report No. Insert if performing organization wishes to assign this number.

10. Work Unit No. Use the agency-wide code (for example, 923-50-10-06-72), which uniquely identifies the work unit under which the work was authorized. Non-NASA performing organizations will leave this blank.

11. Insert the number of the contract or grant under which the report was prepared.

15. Supplementary Notes. Enter information not included elsewhere but useful, such as: Prepared in cooperation with... Translation of (or by)... Presented at conference of. .. To be published in...

16. Abstract. Include a brief (not to exceed 200 words) factual summary of the most significant information contained in the report. If possible, the abstract of a classified report should be unclassified. If the report contains a significant bibliography or literature survey, mention it here.

17. Key Words. Insert terms or short phrases selected by the author that identify the principal subjects covered in the report, and that are sufficiently specific and precise to be used for cataloging.

18. Distribution. Statement. Enter one of the authorized statements used to denote releasability to the public or a limitation on dissemination for reasons other than security of defense information. Authorized statements are "Unclassified-Unlimited, "U.S. Government and Contractors only," "U.S. Government Agencies only, " and "NASA and NASA Contractors only."

19. Security Classification (of report). NOTE: Reports carrying a security classification will require additional markings giving security and downgrading information as specified by the Security Requirements Checklist and the DoD Industrial Security Manual (DoD 5220.22-M).

20. Security Classification (of this page). NOTE: Because this page may be used in preparing announcements, bibliographies, and data banks, it should be unclassified if possible. If a classification is required, indicate separately the classification of the title and the abstract by following these items with either "(U)" for unclassified, or "(C)" or "(S)" as applicable for classified items.

21. No. of Pages. Insert the number of pages.

22. Price. Insert the price set by the Clearinghouse for Federal Scientific and Technical Information or the Government Printing Office, if known. 


\section{RECEDING PAGE BLANK NOT FILAED}

\section{PREFACE}

The work described in this report was performed by the Guidance and Control Division of the Jet Propulsion Laboratory. 


\section{ACKNOW LEDGMENTS}

It is a pleasure to acknowledge the contribution of $\mathrm{Mr}$. William Christensen for his assistance in taking data and $\mathrm{Mr}$. George Anno of ART Associates for performing the Monte Carlo calculations. 


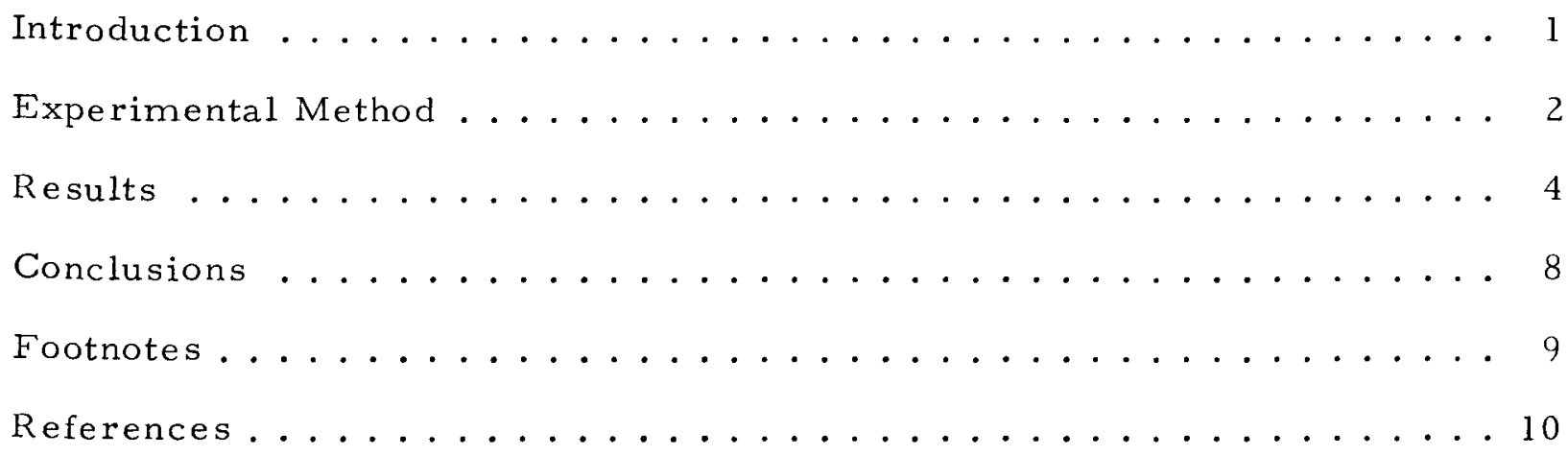

TABLES

I. Efficiency (Counts $/ 10^{3} \gamma$ ) of Aluminum-Covered

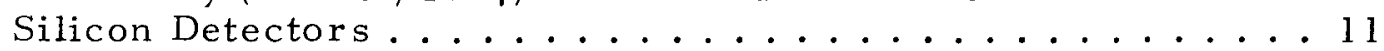

II. Efficiency (Counts/10 $\mathrm{\gamma}$ ) of Lead-Covered

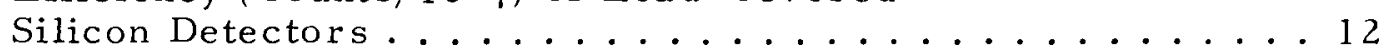

\section{FIGURES}

1. The response of a $0.3-$ and $5-\mathrm{mm}$ aluminumcovered silicon detector to gamma rays........... 13

2. The response of a 0.3 - and $5-\mathrm{mm}$ covered silicon detector at an electron bias of $75 \mathrm{keV} \ldots \ldots \ldots 14$

3. The response of a 0.3 - and $5-\mathrm{mm}$ covered silicon detector at an electron bias of $200 \mathrm{keV} \ldots \ldots \ldots$ 


\begin{abstract}
Measurements have been made of the efficiency in detecting gamma rays of a $0.3-\mathrm{mm}$, a $3-\mathrm{mm}$, and a $5-\mathrm{mm}$ silicon detector covered with different absorbers. Calibrated sources covering the range from $279 \mathrm{keV}$ to $2.75 \mathrm{MeV}$ were used. The need for the absorbers in order to obtain meaningful results and their contribution to the response of the detectors at electron biases from 50 to $200 \mathrm{keV}$ are discussed in detail. It will be shown that the results are virtually independent of the atomic number of the absorber. In addition, the role of the absorber in increasing the efficiency with increasing photon energy for low bias settings is demonstrated for the $0.3-\mathrm{mm}$ crystal. Qualitative explanations are given for the shapes of all curves of efficiency versus energy at each bias.
\end{abstract}


$\underline{\text { Introduction }}$

Silicon detectors are designed mainly for charged-particle work. Numerous measurements and calculations have been made to study their response to electrons ( $\underline{1}-\underline{4})$; however, there seems to have been no comprehensive effort of a similar nature with respect to gamma rays. This report presents the results of an experimental investigation of the response of three covered silicon detectors (0.3-,3-, and 5-mm thick) to monoenergetic gamma rays with energies from $279 \mathrm{keV}$ to $2.75 \mathrm{MeV}$. The covers used were Lucite, aluminum, copper, and lead. The need for a cover and the conclusions which can be drawn from the use of different covers will be discussed. Representative results and their interpretation will be presented here. ${ }^{a}$

Silicon detectors have been used routinely as a laboratory instrument for a number of years. Their advantages over other conventional chargedparticle detectors are significant. The density is high compared with gas counters; the energy required to produce an electron-hole pair is low (3.6 eV/pair); their resolution is very narrow (typically about 25 to $40 \mathrm{keV}$ ) and virtually independent of energy.

They have been used extensively as charged-particle detectors in numerous flights in the U.S. space program. It is almost certain that future long-range flights will use radioisotope thermoelectric generators (RTGs) to provide the power for science packages. These RTGs use $\mathrm{PuO}_{2}$ that contains about $80 \%{ }^{238} \mathrm{Pu}$ as the heat source. The background of the silicon detectors can be expected to rise due to: the gamma rays arising from the decay of ${ }^{238} \mathrm{Pu},{ }^{239} \mathrm{Pu}$, and ${ }^{21} \mathrm{Ne}$ produced in the $\alpha-\mathrm{n}$ reaction in ${ }^{18} \mathrm{O} ;{ }^{22} \mathrm{Ne}$ resulting from the $\alpha-n$ reaction with the impurity ${ }^{19} \mathrm{~F}$; and ${ }^{228}$ Th resulting from the decay of ${ }^{236} \mathrm{Pu}$, an impurity in the fuel. For instance, a 
five-year-old $2000-W$ RTG will emit radiation equivalent to a gamma source of about $8.25 \times 10^{8}$ disintegrations/s in the $700-\mathrm{keV}$ region. In addition, there will be a buildup of ${ }^{228}$ Th with a decay rate of about $1.85 \times 10^{9}$ disintegrations/s. This decays very quickly with gamma emission primarily from ${ }^{212} \mathrm{~Pb},{ }^{212} \mathrm{Bi}$, and ${ }^{208} \mathrm{Tl}$. One may expect that these gamma rays will result in an increase in the background of charged-particle detectors placed in the vicinity of the $R T G$.

\section{Experimental Method}

The electronics consisted of a charge-sensitive preamplifier, amplifier, discriminator, and scaler. The $5.305-\mathrm{MeV} \alpha$ particle of ${ }^{210}$ Po was used for energy calibration. The error resulting from the pulse-height defect in silicon () is negligible for $5.3 \mathrm{MeV} \alpha$ particles.

All the detectors were $2 \mathrm{~cm}^{2}$ in area and were about $25 \mathrm{~cm}$ from the source, with the plane of the silicon normal to the source vector. The detectors were wrapped in several layers of $0.0013 \mathrm{~cm}(0.5 \mathrm{mil})$ aluminized Mylar to exclude light. The $0.3-\mathrm{mm}$ one was fully depleted; the 3 - and 5-mm ones were lithium drifted.

The sources used were ${ }^{203} \mathrm{Hg}\left(\mathrm{E}_{\gamma}=0.279 \mathrm{MeV}\right),{ }^{137} \mathrm{Cs}_{\mathrm{\gamma}}\left(\mathrm{E}_{\gamma}=\right.$ $0.662 \mathrm{MeV}),{ }^{54} \mathrm{Mn}\left(\mathrm{E}_{\gamma}=0.835 \mathrm{MeV}\right),{ }^{60} \mathrm{Co}\left(\mathrm{E}_{\gamma}\right.$, aver $\left.=1.25 \mathrm{MeV}\right)$, and ${ }^{24} \mathrm{Na}\left(E_{Y}=1.37\right.$ and $\left.2.75 \mathrm{MeV}\right)$. They were calibrated with an accuracy of about $3 \%$ using a $\mathrm{Ge}(\mathrm{Li})$ crystal (ㅁ) .

It is virtually impossible to obtain monoenergetic gamma-ray sources of convenient energies with no accompanying electrons. The calibrated sources used in the se measurements were covered with $0.025 \mathrm{~cm}$ Mylar, which is thinner than the range of the electrons emitted. The problem is complicated by the fact that the detectors used are virtually transparent to gamma rays. Electrons, however, cannot traverse the silicon without depositing some energy. There is also the contribution of electrons arising 
from the scattering of gamma rays in the walls and other material in the vicinity of the detector. The accurate evaluation of all these effects is a practical impossibility. For instance, Monte Carlo calculations ${ }^{b}$ show that a change in the internal conversion coefficient of only $0.03 \%$ will increase the counting rate of a $0.3-\mathrm{mm}$ detector by about 10 to $15 \%$. The uncertainty in the measurement of an internal conversion coefficient as small as $0.03 \%$ may be as high as $50 \%$. This can easily cause a prohibitively large error in the sensitivity. The same argument holds for the shape and intensity of the various groups of the $\beta$ spectra. This entire problem can be eliminated by placing an absorber directly in front of the detector. If the absorber is thicker than the range of all electrons originating in the source or created in the room by Compton scattering of the gammas, the detector will be effectively shielded from these electrons. Additional free electrons are created in the absorber, and many of them reach the silicon and contribute to the efficiency; however, the geometry is localized, the uncertainties in the source electron intensities are eliminated, and the physics of gamma interaction and electron transport are the same for the absorber and detector. Thus, no additional complexities are introduced. In addition, if the absorber is thicker than the range of the maximum energy electrons created there, and if a small correction is made for the gamma attenuation in the absorber, the efficiency of the system is independent of the thickness. (This assumes that the diameter is large compared with the thickness.) The efficiency with this technique is due to the combined effects of interaction in the absorber as well as the detector. ${ }^{c}$

Runs were also made with the absorber on both sides of the detector. Although this has no intrinsic value in arriving at an understanding of the physics involved (it is safe to assume that the introduction of a material 
which increases the albedo should, in most cases, raise the counting rate), it simulates more realistically a condition which might be encountered aboard a spacecraft.

$\underline{\text { Results }}$

In most of the cases, the attenuation of the photon beam by the absorber is small. The $279-\mathrm{keV}$ gamma rays of ${ }^{203} \mathrm{Hg}$ on the lead absorber is a notable exception. The data in the tables have been corrected to zero absorber thickness. This assumes an absorber which can stop all source electrons, is thicker than the range of electrons produced at its front surface, but does not attenuate the gamma beam from the source. Although it is an artificial condition, it eliminates the additional effect of gamma shielding, which is of no interest in this paper.

In order to view the data properly, it is essential to consider the absorber as an integral part of the counter. In addition to stopping electrons from the source, it generates free electrons, many of which reach the silicon and are counted. The $0.3-\mathrm{mm}$ silicon detector is thinner than the range - of the maximum energy electrons produced by the gammas in the se measurements, except for the case of ${ }^{203} \mathrm{Hg}$. Monte Carlo calculations show that the absorbers, which are much thicker than the $0.3-\mathrm{mm}$ detector, make a greater contribution to the efficiency than the silicon.

Tables I and II present the measured efficiencies, $\epsilon$, in counts per $10^{3}$ photons incident on aluminum-and lead-covered detectors. The errors include the counting errors, the source calibration errors, and a $2 \%$ error resulting from an assumed $1 \%$ error in distance measurements. The errors at 2. $754 \mathrm{MeV}$ include an additional estimated error of about $3 \%$, resulting from the subtraction of the efficiency of the $1.37-\mathrm{MeV}$ gamma of ${ }^{24} \mathrm{Na}$. The measurements performed by Endres, et al., (ㅇ) were very similar to 
those described in this report and need no elaboration. The errors in their values are about 5\%. Figure 1 gives the $0.3-$ and $5-\mathrm{mm}$ data of $\epsilon$ versus $E$ at different biases for aluminum covers. The curves for the other covers exhibit the same general behavior. Figures 2 and 3 show $\epsilon$ for the $0.3-$ and $5-\mathrm{mm}$ at 75 and $200 \mathrm{keV}$ bias for all absorbers. (Corresponding plots of the 3-mm data are very similar to the 5-mm results and are not shown. Figures 1 to 3 show the data for the sandwiched detectors only.)

Several features of the $0.3-\mathrm{mm}$ results are immediately obvious in Fig. 1. The efficiency increases rapidly with energy except for the $200-\mathrm{KeV}$ bias case where it ultimately becomes flat. The results at lower biases are readily understandable. As the gamma-ray energy increases, the Compton electron energy increases. The electrons can penetrate a greater thickness of absorber. The detector, which includes the absorber, has a larger effective volume and more electrons generated in the absorber can reach the silicon. This enhancement of the efficiency more than compensates for the reduction of the Compton cross section. The fact that the curves ultimately get flat for the $200-\mathrm{keV}$ bias is probably the result of more subtle effects. More of the absorber can be considered as a part of the counter as the energy increases from 1.25 to $2.75 \mathrm{MeV}$; however, the angular distribution of Compton electrons is peaked much more in the forward direction at 2. $75 \mathrm{MeV}$. This means that a larger fraction of electrons enter the silicon in a near-normal direction. Monte Carlo calculations performed for $500-\mathrm{keV}$ electrons entering a $0.3-\mathrm{mm}$ silicon detector in a normal direction show that less than $20 \%$ of the electrons lose more than $200 \mathrm{keV}$ of energy in the detector. This fraction would be much smaller for forward scattered electrons from a $2.75-\mathrm{MeV}$ photon than a $1.25 \mathrm{MeV}$ source. A large percentage of electrons created in the side of the aluminum close to the 
detector would not deposit $200-\mathrm{keV}$ energy in the silicon. In addition, fewer of the Compton events in the silicon would produce a count at a $200-\mathrm{keV}$ bias.

The absorber on the back side of the detector produces several

effects. On the one hand, it prevents free electrons created in the room from entering the back side of the detector. On the other hand photons from the source incident on the back absorber can create electrons that may get into the detector. If the interaction is a Compton event, the electrons will be produced in a forward direction (away from the detector) and will probably not be detected. (A small percentage will be reflected from the back absorber to the detector.) If the event is a photoelectric or pair process, we can expect more electrons in the backward direction (toward the detector) and an enhancement of the efficiency. This effect is seen in Table II with the lead absorber. It is less prominent in the aluminum and lucite absorber. It is seen in the copper absorber mainly at $2.75 \mathrm{MeV}$.

A striking feature discernible from an inspection of Figures 2 and 3 is the relative independence of response on the atomic number of the cover. This result is not surprising. In addition to shielding electrons emitted by the source, all covers were made thicker than the range of the maximum energy electrons that could be produced by photon interactions in the absorber. For all cases, except lead, the only electron production process of any consequence is by Compton collision. The cross section for this is proportional to the atomic number, Z. The expression for electron energy loss is very close to being proportional to $Z$, i.e., $\approx Z \ln 1 / Z$. The probability of a photon interacting in the absorber to produce an electron which reaches the detector is proportional to $\sigma_{\text {comp }} /(d E / d x)$, which is only very weakly dependent on $\mathrm{Z}$. Lead is an exception because the probability of the photoelectric or pair process in lead for the gamma-ray energies used in 
these measurements is not negligible and the cross section for both of these interactions involve higher powers of $\mathrm{Z}$. Indeed, the response for lead covers is generally higher than the others.

The shape of the curves for the thick crystals is very easily understood. As in the $0.3-\mathrm{mm}$ case, the efficiency is almost entirely independent of the $\mathrm{Z}$ of the cover. In the $3-$ and $5-\mathrm{mm}$ case, however, the crystal has a much greater effect than the cover in its contribution to the overall efficiency of the combination. Most of the counts are due to interactions that occur in the crystal, which is thick compared with the distance required for an electron to travel in order to lose $200 \mathrm{keV}$ of energy. As a result the efficiency is virtually independent of the photon energy at every bias. The argument, used in the $0.3-\mathrm{mm}$ case, that the cover appears thicker as we increase the average energy of electrons resulting from interactions in the cover, is still valid; however, this contribution relative to free electrons created in the silicon, is reduced as the silicon gets thicker. Monte Carlo calculations demonstrate explicitly the diminishing contribution of the cover to the total efficiency.

If

$$
\begin{aligned}
\mathrm{E}_{\mathrm{Si}} \equiv & \text { the energy absorbed in the -silicon from electrons } \\
& \text { originating in the silicon } \\
\mathrm{E}_{\mathrm{t}} \equiv & \text { the energy absorbed in the silicon from electrons } \\
& \text { originating in the silicon or the absorber }
\end{aligned}
$$

then

$$
\begin{aligned}
& E_{S i} / E_{t} \simeq 0.1 \text { for the } 0.3-\mathrm{mm} \text { crystal at } E_{\gamma}=0.835 \mathrm{MeV} \\
& E_{S i} / E_{t} \simeq 0.9 \text { for the } 5-\mathrm{mm} \text { crystal at } E_{\gamma}=1.25 \mathrm{MeV}
\end{aligned}
$$

The value at the 279-keV gamma energy falls off abruptly, except for the 50-keV bias, because there are few Compton electrons which exceed the bias for $E_{Y}=279 \mathrm{keV}$. 


\section{Conclusions}

The following conclusions may be drawn from this investigation:

(1) Meaningful results on the efficiency of silicon detectors for gamma rays cannot be obtained unless source electrons can be excluded from the measurement.

(2) The covers must be thick enough to absorb all source electrons and the most energetic electrons created by gamma interaction on the front surface of the cover. If the latter restriction is violated, the data will be sensitive to the thickness of the cover.

(3) The efficiency is very insensitive to the $Z$ of the cover.

(4) If most of the counts are due to free electrons created in the cover, the efficiency increases with gamma-ray energy unless the bias is greater than the energy electrons lose in traversing the crystal. In the latter case a saturation effect ultimately is seen as the photon energy is increased. If most of the counts are due to free electrons which originate in the silicon and if the silicon is thick compared with the bias energy, then this efficiency saturation occurs at much lower gamma-ray energies. 
Eootnotes

${ }^{\mathrm{a}}$ The complete results are available and may be obtained from the author.

$\mathrm{b}$

All Monte Carlo calculations referred to in this paper were performed using the BETA code ( $\underline{7})$.

${ }^{c}$ This introduces a problem involving the source-to-detector distance. The problem is trivial for a bare silicon detector which is thick compared with the bias energy. In that case the center of detection is at the center of the crystal. The center of detection of a system which includes the cover and silicon can be determined by Monte Carlo techniques; however, it was arbitrarily placed at the front surface in the $0.3-\mathrm{mm}$ case and at the center of the crystal for the two thicker cases. The error should be small because the thickness of the entire detector system (cover plus crystal) was small compared with the source-to-detector distance. 


\section{References}

1. M. J. BERGER, et al., Nucl. Instr. and Meth., 69, 181 (1969).

2. J. M. McKENZIE and G. T. EWAN, IRE Trans. Nucl. Sci., NS-8, No. $1,50(1961)$.

3. H. I. WEST, et al., Rev. Sci. Instr., 33, 380 (1962).

4. P. CHAROENKWAN, Nucl. Instr, and Meth., 34, 93 (1965).

5. MOJTABA TAHERZADEH, personal communication.

6. M. REIER, Nucl. Sci. and Eng. 43, 267 (1971).

7. T. M. JORDAN, "BETA, a Monte Carlo Computer Program for Bremsstrahlung and Electron Transport Analysis, "AFWL-TR-68-111, Air Force Weapons Laboratory, Kirtland Air Force Base (1968).

8. G. W. R. ENDRES, et al., "Study of Interaction of Low Dose Power Source Radiation Fields with Selected Space Scientific Instruments," Y-49607, Battelle Memorial Institute, Richland, Wash. (1970). 


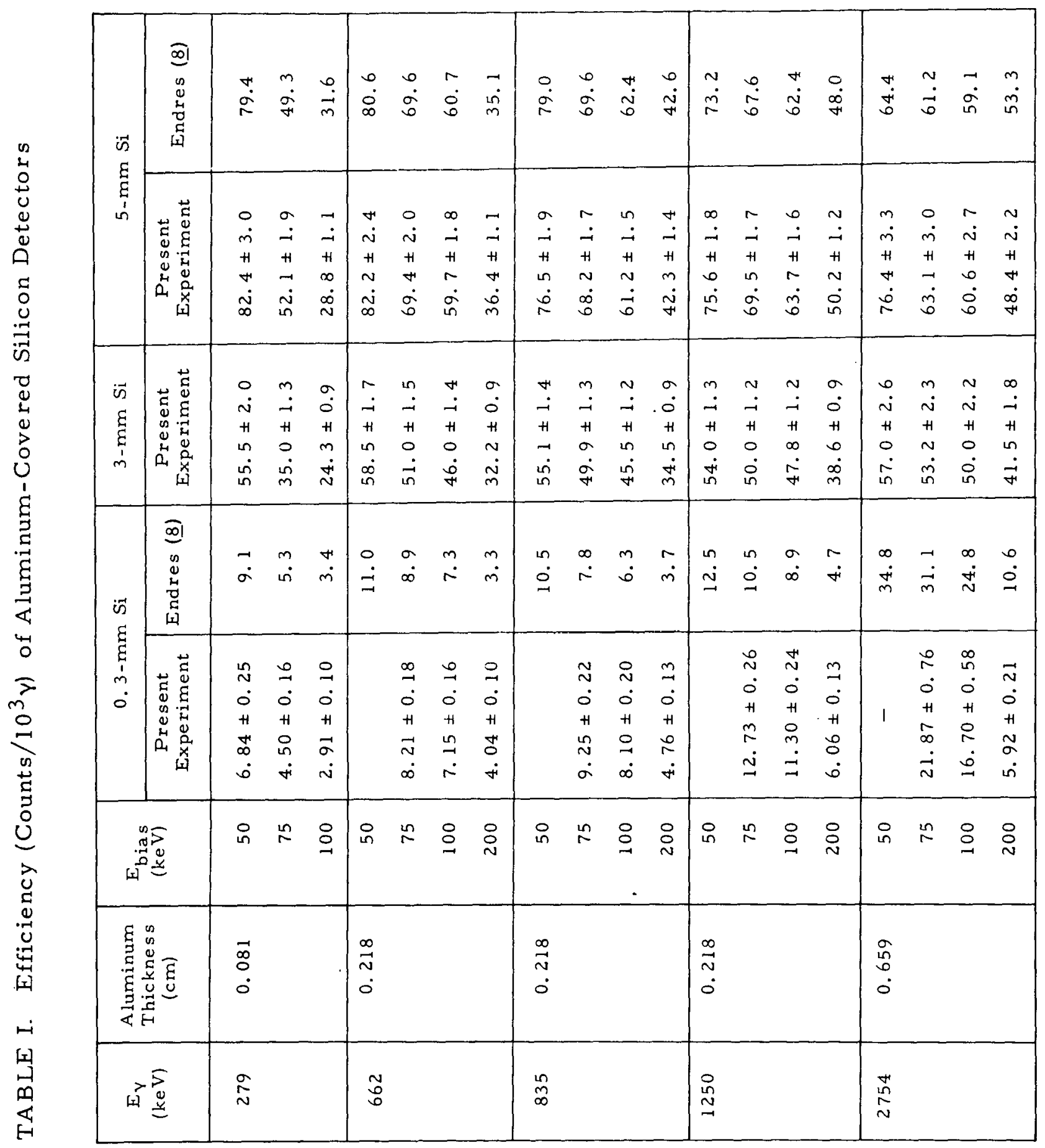




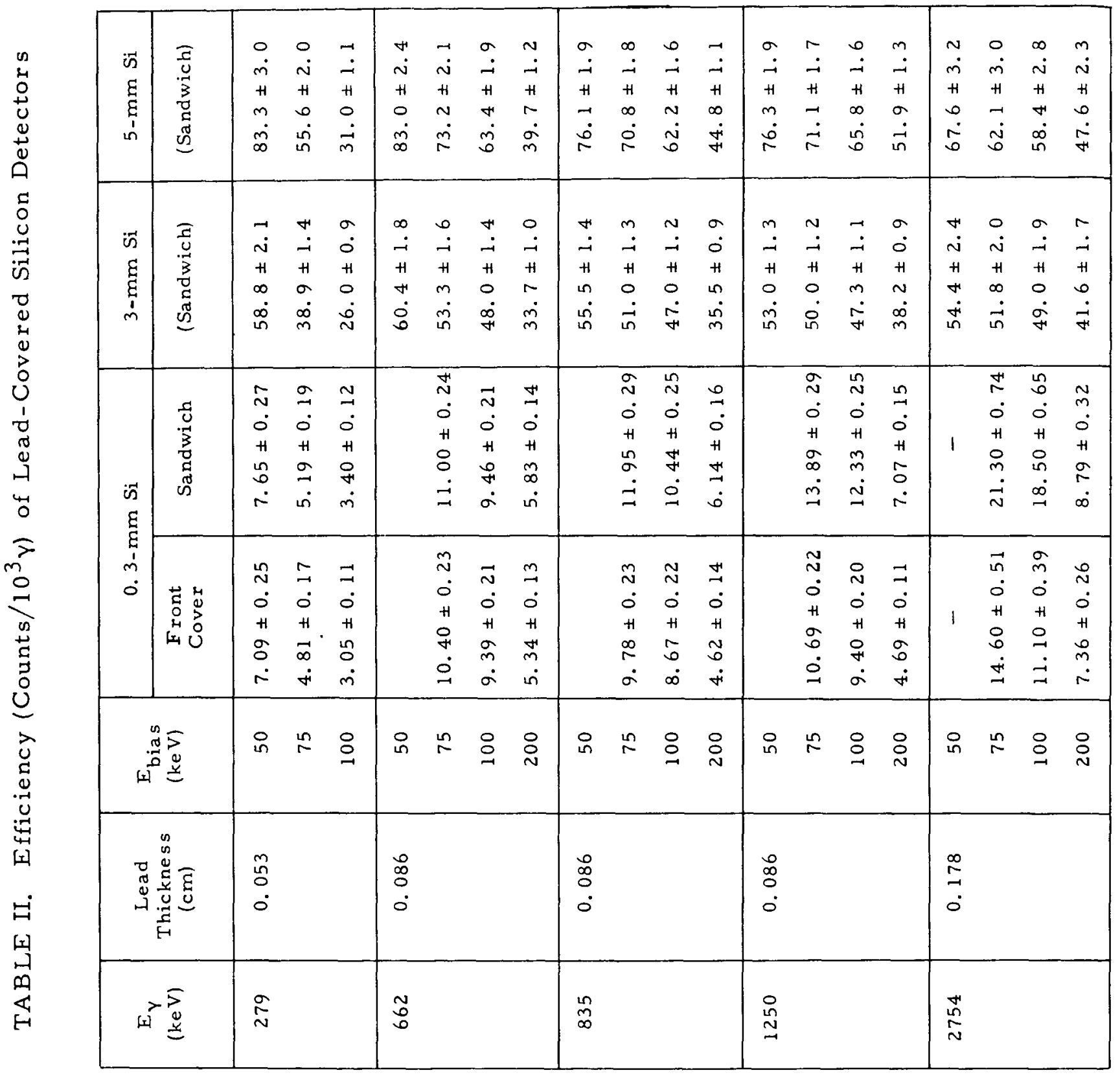




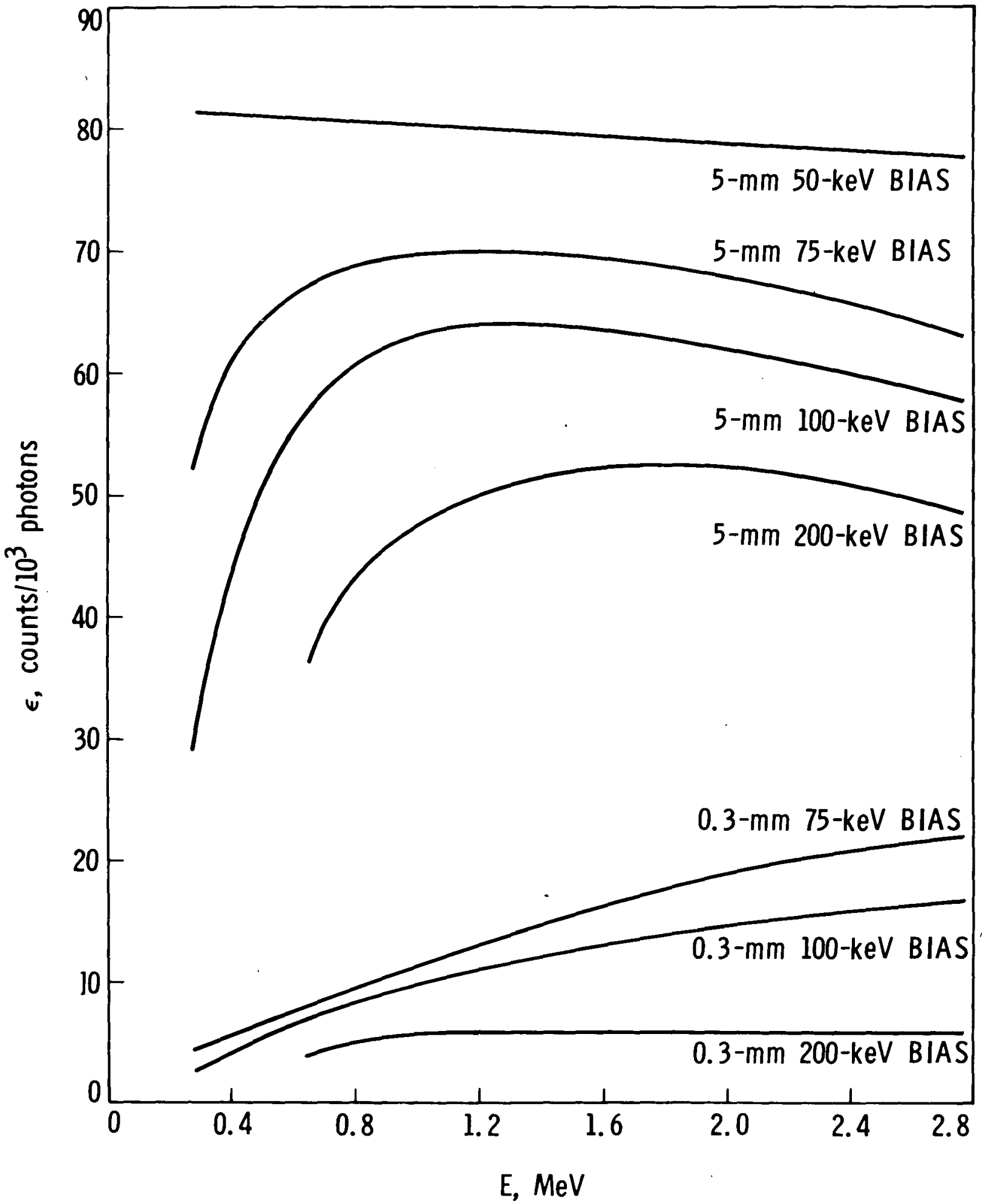

Fig. 1. The response of a $0.3-$ and $5-\mathrm{mm}$ aluminum-covered silicon detector to gamma rays 


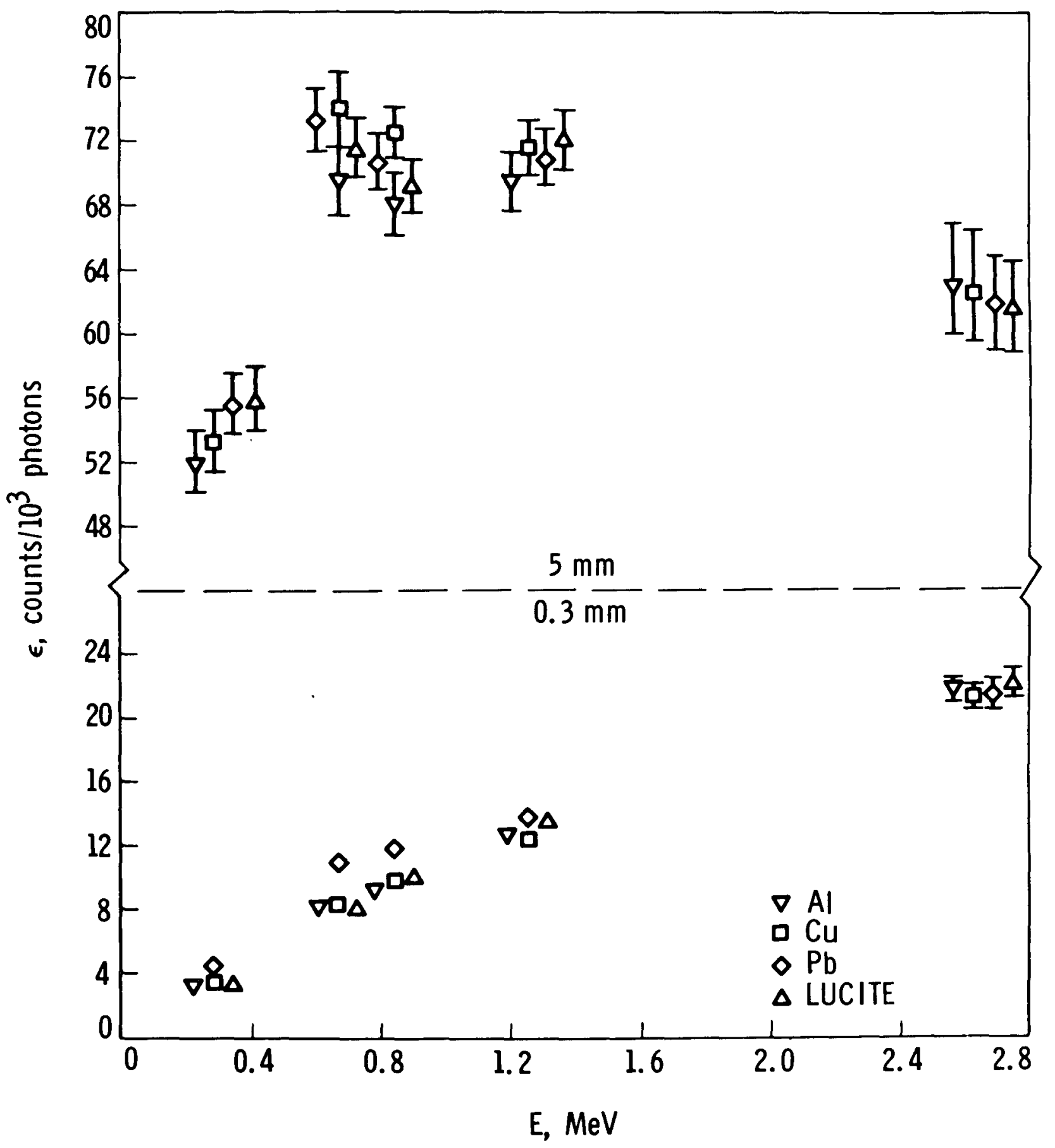

Fig. 2. The response of a $0.3-$ and 5-mm covered silicon detector at an electron bias of $75 \mathrm{keV}$ 


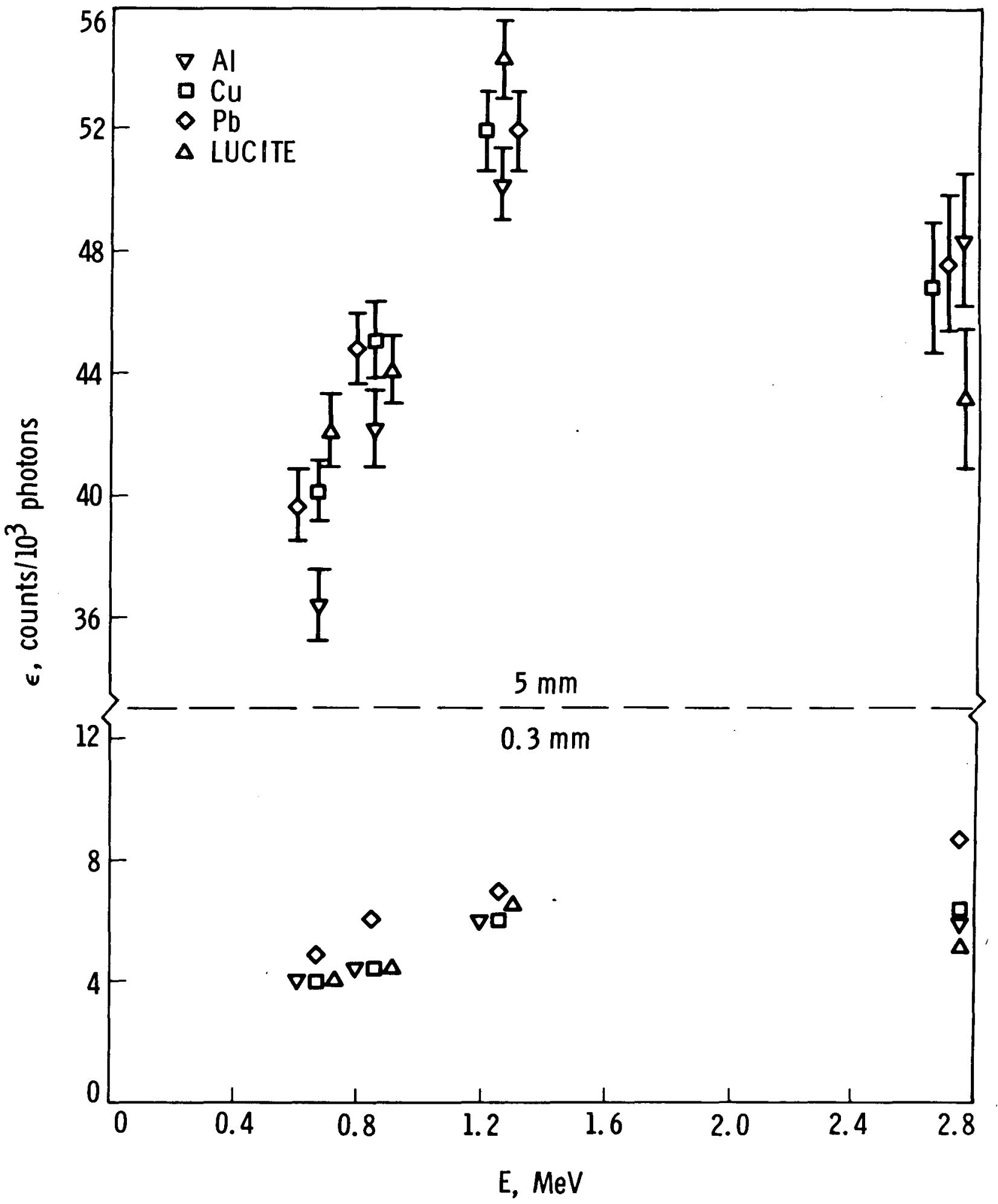

Fig. 3. The response of a $0.3-$ and $5-\mathrm{mm}$ covered silicon detector at an electron bias of $200 \mathrm{keV}$ 